\title{
Model for the magnetoresistance and Hall coefficient of inhomogeneous graphene
}

\author{
Rakesh P. Tiwari and D. Stroud \\ Department of Physics, Ohio State University, Columbus, Ohio 43210, USA \\ (Received 8 December 2008; revised manuscript received 24 February 2009; published 7 April 2009)
}

\begin{abstract}
We show that when bulk graphene breaks into $n$-type and $p$-type puddles, the in-plane resistivity becomes strongly field dependent in the presence of a perpendicular magnetic field even if homogeneous graphene has a field-independent resistivity. We calculate the longitudinal resistivity $\rho_{x x}$ and Hall resistivity $\rho_{x y}$ as a function of field for this system using the effective-medium approximation. The conductivity tensors of the individual puddles are calculated using a Boltzmann approach suitable for the band structure of graphene near the Dirac points. The resulting resistivity agrees well with experiment provided that the relaxation time is weakly field dependent. The calculated Hall resistivity has the sign of the carriers in the puddles occupying the greater area of the composite and vanishes when there are equal areas of $n$ - and $p$-type puddles.
\end{abstract}

DOI: 10.1103/PhysRevB.79.165408

PACS number(s): 72.20.My, 75.47.-m, 73.50.Jt, 72.80.Ng

\section{INTRODUCTION}

Graphene is a two-dimensional form of carbon with a hexagonal crystal structure like that of a single layer of graphite. Because of this structure, it has the band structure of a semimetal: the Fermi energy $E_{F}$ of neutral graphene lies at a "Dirac point," where the electronic density of states $n\left(E_{F}\right)=0$. There are two inequivalent Dirac points located at different Bloch vectors $\mathbf{k}_{0}$ and $\mathbf{k}_{1}$. Near the Dirac points, the bands are linear functions of the components of $\mathbf{k}-\mathbf{k}_{0}$ and $\mathbf{k}-\mathbf{k}_{1}$, and $n(E)$ is proportional to $\left|E-E_{F}\right|$. Because of this unusual band structure, the quasiparticle Hamiltonian near the Dirac points is formally identical to that of massless Dirac fermions, a feature which is responsible for part of the recent interest in graphene.

Graphene also has striking transport properties. For example, experiments have observed finite conductivity for all values of $E_{F}$, whether above or below the Dirac point, ${ }^{1}$ with a minimum conductivity typically $\sim 4 e^{2} / h$. However, some workers have suggested that this minimum could have much smaller $^{2}$ or larger ${ }^{3}$ values than $4 e^{2} / h$. It has been proposed that the existence of a finite conductivity even at the charge neutrality point might be a result of local potential fluctuations, which could cause a homogeneous neutral graphene sheet to break up into "puddles" of electron-rich ( $n$-type) and hole-rich ( $p$-type) characters. ${ }^{4}$ These puddles have, in fact, been unambiguously observed in experiments using scanning tunneling microscopy. ${ }^{5}$

Recently measurements of the magnetic-field-dependent longitudinal and Hall resistivity $\rho_{x x}$ and $\rho_{x y}$ measurements have been reported. ${ }^{6} \rho_{x x}$ was found to increase by nearly tenfold with increasing magnetic field perpendicular to the graphene film, followed by an apparent saturation at sufficiently strong magnetic field. These authors found that the magnetoresistance was inconsistent with a two-fluid model of transport by $n$-type and $p$-type charge carriers in a homogeneous sheet of graphene and suggested that it might agree better with a model describing the film as a mixture of $n$-type and $p$-type puddles. However, they were able to obtain close agreement between experiment and theory only by assuming an ad hoc empirical form for the magnetoresistance.

In this paper, we present a simple model for the magnetoresistance and Hall coefficients of graphene based on the effective-medium approximation (EMA) in a transverse magnetic field. Such a model is reasonable if the $n$-type and $p$-type puddles are distributed randomly, as appears to be the case in Ref. 6. Our results show that when the area fractions of $n$-type and $p$-type puddles are exactly equal, $\rho_{x x}$ varies exactly linearly with field. At other puddle fractions, it saturates, in agreement with experiment. We find that we can obtain excellent agreement with the observed behavior of $\rho_{x x}(B)$ if we assume an $n$-type area fraction $f_{n}$ satisfying $\left|f_{n}-1 / 2\right| \sim 0.07$ and reasonable values for average carrier density and transport relaxation time. We also make predictions about the Hall resistivity $\rho_{x y}(B)$.

\section{MODEL}

We consider magnetotransport in a single layer of graphene subject to a magnetic field $\mathbf{B}=B \hat{z}$ perpendicular to the graphene layer. We assume that, because of a random potential due to charges in the substrate or some other cause, the graphene layer has broken up into a mixture of $n$-type and $p$-type puddles, having area fractions $f_{n}$ and $f_{p}=1-f_{n}$. We also assume that each of the puddles is large enough to be described by its own magnetoconductivity tensor $\sigma_{n}$ or $\sigma_{p}$. In practice, this assumption means that the puddle dimensions are larger than a typical carrier mean free path. If this condition is not satisfied, the results below would need to be modified.

\section{A. Conductivity tensors of individual puddles}

We assume that $\sigma_{n}$ and $\sigma_{p}$ are both given by the usual free-electron (or free-hole) forms, suitably modified to account for the linear dispersion relations of the electrons and holes near the Dirac point. We denote the charge-carrier densities in the $n$-type and $p$-type puddles by $n$ and $p$, and the corresponding relaxation times by $\tau_{n}$ and $\tau_{p}$. We also initially assume that all the puddles have the same density of charge carriers so that $n=p$ and that the relaxation times $\tau_{n}=\tau_{p} \equiv \tau$. With these assumptions, the zero-field conductivities $\sigma_{n, 0}$ and $\sigma_{p, 0}$ of the $n$-type and $p$-type puddles are equal $\left(\sigma_{n, 0}\right.$ $\left.=\sigma_{p, 0} \equiv \sigma_{0}\right)$. We can also define zero-field mobilities $\mu_{n}$ and $\mu_{p}$ by $\sigma_{n}=n e \mu_{n}$ and $\sigma_{p}=p e \mu_{p}$, where $e$ is the magnitude of 


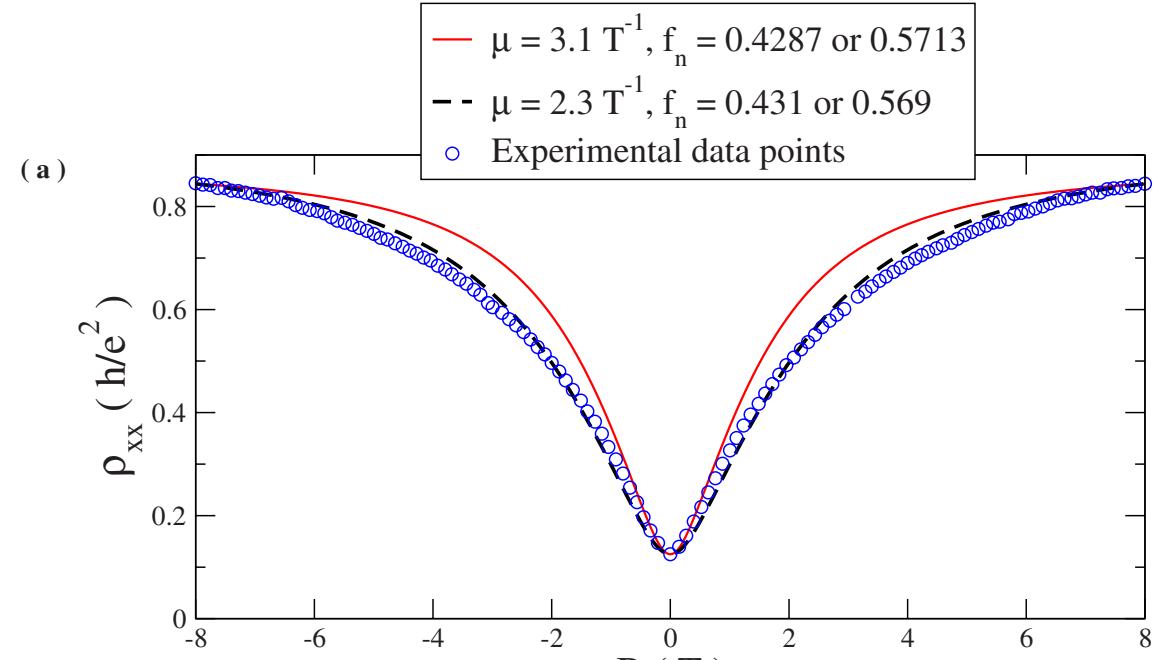

( b )

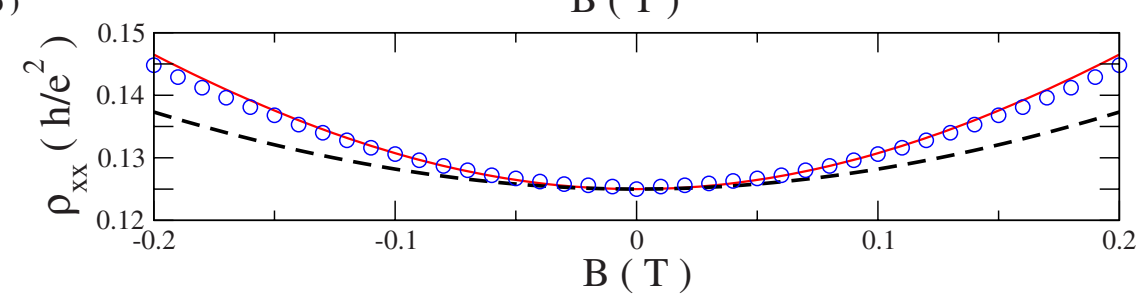

FIG. 1. (Color online) $\rho_{x x}\left(B, f_{n}\right)$ as a function of $B$ (in T), with two different assumptions about the mobility. In both cases, we assume that the electron and hole mobilities are equal. Solid (red) line: calculated results, assuming $\mu \equiv \omega_{c} \tau / B=3.1 \mathrm{~T}^{-1}$ and $f_{n}=0.429$ (or 0.571). Dashed (black) line: calculated results with $\mu=2.3 \mathrm{~T}^{-1}$ and $f_{n}=0.431$ (or $0.569)$. Open circles are experimental data from Ref. 6. Lower panel is a blowup of the calculations and data from the upper panel. the electronic charge; with the above assumptions, these mobilities are also equal $\left(\mu_{n}=\mu_{p} \equiv \mu\right)$. Later in this section, we will consider the more general cases where $n \neq p$ or $\tau_{n} \neq \tau_{p}$.

With these assumptions, the $2 \times 2$ conductivity tensor in the $x y$ plane takes the form

$$
\sigma_{n}=\sigma_{n, 0}\left[\begin{array}{cc}
\frac{1}{1+\left(\omega_{c, n} \tau_{n}\right)^{2}} & \frac{\omega_{c, n} \tau_{n}}{1+\left(\omega_{c, n} \tau_{n}\right)^{2}} \\
-\frac{\omega_{c, n} \tau_{n}}{1+\left(\omega_{c, n} \tau_{n}\right)^{2}} & \frac{1}{1+\left(\omega_{c, n} \tau_{n}\right)^{2}}
\end{array}\right]
$$

and

$$
\sigma_{p}=\sigma_{p, 0}\left[\begin{array}{cc}
\frac{1}{1+\left(\omega_{c, p} \tau_{p}\right)^{2}} & -\frac{\omega_{c, p} \tau_{p}}{1+\left(\omega_{c, p} \tau_{p}\right)^{2}} \\
\frac{\omega_{c, p} \tau_{p}}{1+\left(\omega_{c, p} \tau_{p}\right)^{2}} & \frac{1}{1+\left(\omega_{c, p} \tau_{p}\right)^{2}}
\end{array}\right] .
$$

Both the zero-field conductivities $\sigma_{n, 0}$ and $\sigma_{p, 0}$ of the puddles and the cyclotron frequencies $\omega_{c, n}$ and $\omega_{c, p}$ are modified from their usual free-electron values because of the linear dispersion relations near the Dirac point. The result for $\sigma_{n, 0}$ and $\sigma_{p, 0}$ at temperature $T=0$ (fully degenerate limit) is

$$
\sigma_{n, 0}=\frac{2 e^{2}}{h} v_{F} \tau_{n} \sqrt{\pi n}
$$

and

$$
\sigma_{p, 0}=\frac{2 e^{2}}{h} v_{F} \tau_{p} \sqrt{\pi p}
$$

where $v_{F}$ is the Fermi velocity (which is the same for both electrons and holes). This form is obtained from the usual solution of the Boltzmann equation for a degenerate Fermi gas, ${ }^{7}$ which gives for electronic conductivity at zero magnetic field,

$$
\sigma_{n, 0}=\left[2 e^{2} \tau_{n} /\left(2 \pi^{2}\right)\right] \int d^{2} k^{\prime} \hbar^{-2}\left[\partial E\left(\mathbf{k}^{\prime}\right) / \partial k_{x}^{\prime}\right]^{2} \delta\left[E\left(\mathbf{k}^{\prime}\right)-E_{F}\right] .
$$

Here, $\mathbf{k}^{\prime}$ is the two-dimensional wave vector, measured relative to one of the Dirac points, and $E\left(\mathbf{k}^{\prime}\right)=\hbar v_{F}\left|\mathbf{k}^{\prime}\right|$ is the energy relative to the Dirac point. The hole conductivity is given by a similar expression. We use $k_{F, n}=\sqrt{\pi n}$, which takes into account the two valleys near the two inequivalent Dirac points in the graphene band structure, and we have included an extra factor of 2 in Eq. (5) for the same reason. $k_{F, p}$ is given by an analogous expression.

The cyclotron frequency $\omega_{c, n}$ is readily obtained from the semiclassical equation of motion $\hbar \dot{\mathbf{k}}=e \mathbf{v}_{\mathbf{k}} \times \mathbf{B}$, where $\mathbf{v}_{\mathbf{k}^{\prime}}$ $=\hbar^{-1} \nabla_{\mathbf{k}^{\prime}} E\left(\mathbf{k}^{\prime}\right)$, as applied to a band with the dispersion relation $E\left(\mathbf{k}^{\prime}\right)=v_{F} \hbar\left|\mathbf{k}^{\prime}\right|$; the result is (in SI units)

$$
\omega_{c, n}=\frac{v_{F} e B}{\hbar \sqrt{\pi n}},
$$

where $\omega_{c, p}$ is given by an analogous expression. ${ }^{8}$

Transport in graphene is also affected by the dependence of $\tau_{n}$ or $\tau_{p}$ on physical parameters such as $n, p$, and $B$. It has been suggested ${ }^{4}$ that $\tau$ is proportional to $1 / \sqrt{n}$ (or $1 / \sqrt{p}$ ) for scattering by impurities with short-range potentials but is proportional to $\sqrt{n}$ (or $\sqrt{p}$ ) for impurities with Coulomb potentials. If we assume the latter, then from Eqs. (3) and (4) $\sigma_{0, n} \propto n$ and $\sigma_{0, p} \propto p$ and both $\omega_{c, n} \tau_{n}$, and $\omega_{c, p} \tau_{p}$ are independent of carrier density, as in conventional semiconductors. In our calculations below, we give results using both assumptions about the carrier density dependence of $\tau_{n}$ and $\tau_{p}$. 


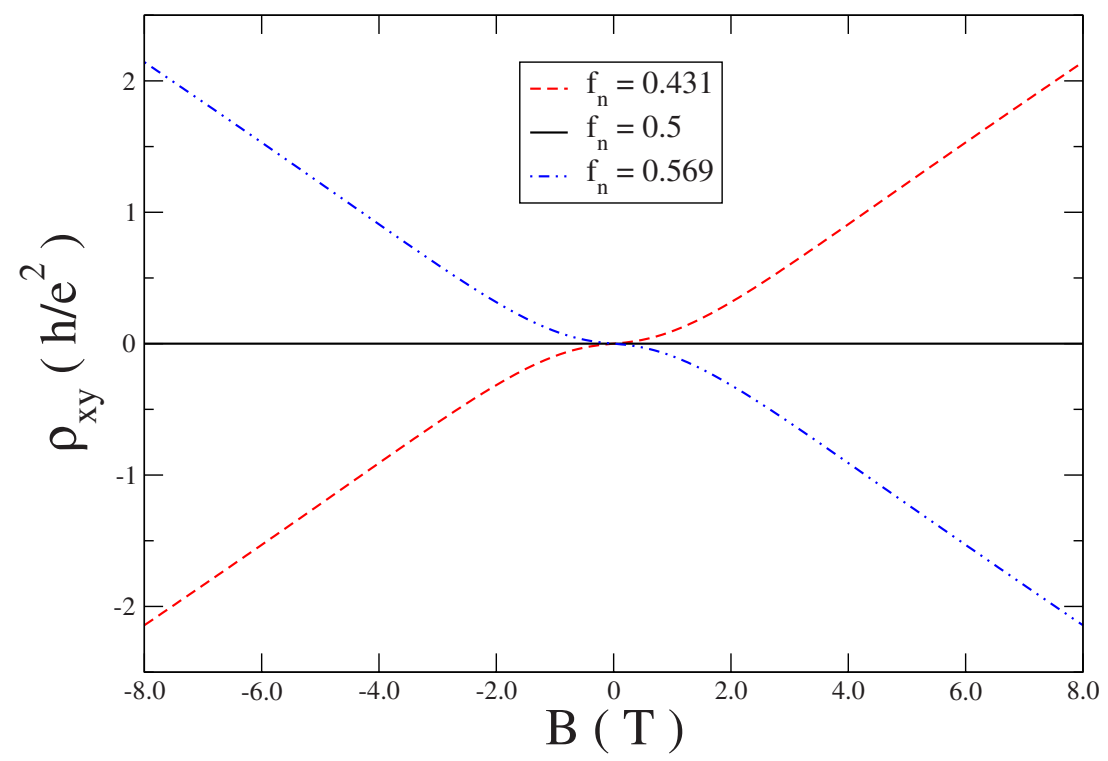

FIG. 2. (Color online) Calculated $\rho_{x y}\left(B, f_{n}\right)$, as obtained in the EMA, using $\mu=2.3 \mathrm{~T}^{-1}$ and $f_{n}$ $=0.431,0.569$, and 0.5 .

\section{B. Effective conductivity of composite of $\boldsymbol{n}$-type and $p$-type puddles}

Next, we calculate the effective conductivity tensor $\sigma_{e}$ of a graphene sheet which has broken up into $n$-type and $p$-type puddles. If $f_{n}=1 / 2$ and $n=p$, this would correspond to the case where the net charge-carrier density is zero, corresponding to a neutral graphene sheet which would, if homogeneous, have its Fermi energy at the Dirac point. However, it is also possible to have a graphene sheet with $f_{n} \neq 1 / 2$ or $n$ $\neq p$, or both, corresponding to a graphene sheet with a net doping. This situation could be produced in a graphene sheet biased by a suitable gate voltage.

A reasonable way of calculating $\sigma_{e}$ for tensor conductivities is provided by the EMA. ${ }^{9}$ In this approach, the electric fields and currents within the inhomogeneous graphene sheet are calculated as if the $n$-type and $p$-type puddles are compact and approximately circular and are embedded in an effective medium whose conductivity is calculated self-consistently.,10 For tensor conductivities, the defining equation for the EMA is

$$
\sum_{i=\{n, p\}} f_{i} \delta \sigma_{i}\left(I-\Gamma \delta \sigma_{i}\right)^{-1}=0 .
$$

Here $\delta \sigma_{i}=\sigma_{i}-\sigma_{e}$ and $I$ is the $2 \times 2$ unit matrix, and for the planar geometry considered, $\Gamma=-I /\left(2 \sigma_{e, x x}\right)$ is the depolarization tensor. This matrix equation reduces to two coupled scalar algebraic equations for the two independent components of $\sigma_{e}\left(\sigma_{e, x x}\right.$ and $\left.\sigma_{e, x y}\right)$, which are easily solved numerically. The other two components are determined by $\sigma_{e, y y}=\sigma_{e, x x}$ and $\sigma_{e, y x}=-\sigma_{e, x y}$. The resistivity tensor is then obtained by inverting the matrix $\sigma_{e}$ so that $\rho_{e, x x}=\rho_{e, y y}=\sigma_{e, x x} /\left(\sigma_{e, x x}^{2}+\sigma_{e, x y}^{2}\right)$ and $\rho_{e, x y}=-\rho_{e, y x}=-\sigma_{e, x y} /\left(\sigma_{e, x x}^{2}+\sigma_{e, x y}^{2}\right)$.

We briefly discuss the conditions under which the EMA is likely to be accurate. As is well known, ${ }^{11}$ the EMA is derived by assuming that, when an electric-field $\mathbf{E}_{0}$ is applied to the composite of $n$ and $p$ puddles, the field $\mathbf{E}_{\text {in }}$ inside any given puddle is equal to that which would be found if the medium outside the puddle is uniform and has an effective conductivity tensor $\sigma_{e}$. The field inside the $i$ th puddle is then found to equal

$$
\mathbf{E}_{\mathrm{in}, i}=\left(I-\Gamma \delta \sigma_{i}\right)^{-1} \mathbf{E}_{0},
$$

where $\Gamma$ is the depolarization tensor (given above for circular puddles). $\sigma_{e}$ is then determined by the self-consistency condition that the space average of the electric field $\mathbf{E}_{\mathrm{in}, i}$ shall equal $\mathbf{E}_{0}$, which leads to Eq. (7). Thus, one might expect that the EMA would be most accurate if the surroundings of a given puddle do not deviate too greatly from the average. However, it is difficult to give a more precise statement of the conditions under which the EMA can be expected to be a good approximation.

The EMA is known to be exact in two limiting cases. First, when the area fraction of $n$ - or $p$-type puddles is small, the EMA becomes exact to first order in $f_{n}$ or $f_{p}$ for circular inclusions of $n$ - or $p$-type conductor in a host with carriers of the opposite $\operatorname{sign}^{12}$ (or, indeed, for a host with any conductivity tensor). Second, the EMA is also exact at $f_{n}=1 / 2$ provided $\omega_{c, p} \tau_{p}=\omega_{c, n} \tau_{n}{ }^{10}$ Because the EMA becomes exact in these two regimes, it seems reasonable to suppose that it will be a good interpolation scheme at other values of $f_{n}$.

Next, we discuss the assumption that the puddles are circular. Clearly, this is at best a rough approximation. Still, one can imagine a "cellular" composite made up of compact roughly circular regions of $n$ - and $p$-type puddles. In this case, it seems reasonable to approximate the electric field inside a compact puddle using Eq. (8). If so, the EMA form [Eq. (7)] is appropriate.

Does the observed puddle morphology of inhomogeneous graphene resemble a cellular composite of compact $n$ - and $p$-type puddles? The published data, obtained using a scanning single electron transistor, ${ }^{5}$ seem to suggest extended, possibly percolating structures of $n$ - and $p$-type regions rather than circular regions. But this picture can be qualitatively reconciled with the random compact puddle picture provided that the area fraction of $n$ and $p$ regions is nearly equal. In this case, according to standard models of 


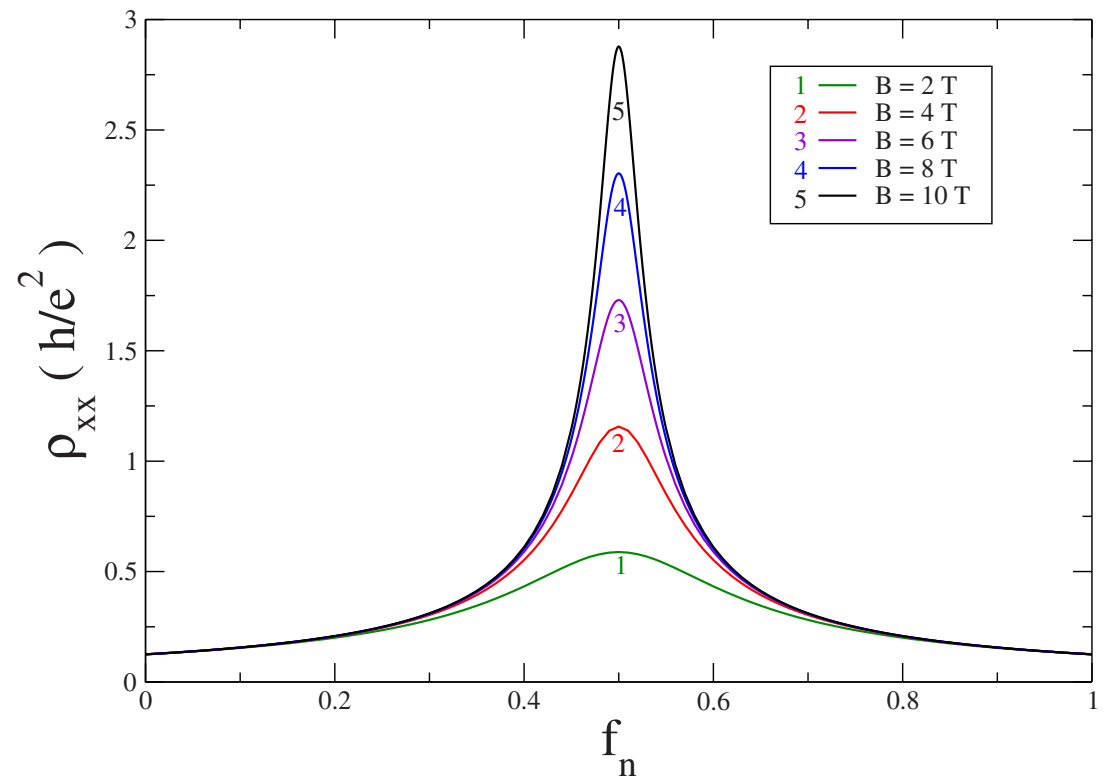

FIG. 3. (Color online) $\rho_{x x}\left(B, f_{n}\right)$ as a function of $f_{n}$ for several values of $B$ and $\mu$ $=2.3 \mathrm{~T}^{-1}$.

percolation, ${ }^{13}$ even if the $n$ - and $p$-type puddles are distributed randomly, they will connect up near percolation to form extended structures similar the experimental pictures. Thus, it may be reasonable to model the observed structures as a distribution of compact $n$ - and $p$-type puddles, as done here.

\section{NUMERICAL RESULTS}

We begin by evaluating the predictions of the above model for the simplest case: $n=p, \tau_{n}=\tau_{p} \equiv \tau$, and $\tau$ independent of $n$. In order to compare this model to experiment, ${ }^{6}$ we need the values of $v_{F}, n, \tau$ (or equivalently, $\mu$ ), and $f_{n}$. From the band structure of graphene, $v_{F} \sim 10^{6} \mathrm{~m} / \mathrm{s} .{ }^{1,14}$ In fact, a value of $1.1 \times 10^{6} \mathrm{~m} / \mathrm{s}$ has been inferred from measurements of the Landau-level splitting in graphene, ${ }^{15}$ and we use this value in the calculations below. Also, the measured value of the zero-field resistivity is $\sigma_{0}^{-1}=\rho_{0}=0.125 h / e^{2}$. Given this value, Eq. (3) provides one condition satisfied by the two parameters $n$ and $\tau$. We then choose $n, \tau$, and $f_{n}$ so as to best fit the measured $\rho_{x x}(B)$ at $B=8 \mathrm{~T}$ and to yield $\omega_{c} \tau=3.1 B$, where $B$ is the magnetic field in T, as reported in Ref. 6 . This procedure gives $n \sim 6 \times 10^{14} \mathrm{~m}^{-2}$ and $f_{n} \sim 0.43$. We find that the best agreement with the resistivity is given at high fields by $\omega_{c} \tau \sim 2.3 B$, and at low fields by $\omega_{c} \tau \sim 3.1 B$, indicating a weakly field-dependent $\tau$. The value of $n$ is close to measured value quoted in Ref. 6.

The calculated results for $\rho_{x x}(B)$ are shown in Fig. 1 using these parameters. As can be seen, the fit to the experimental data is excellent over most of the field range, using $\omega_{c} \tau$ $\sim 2.3 B$, and at low fields using $\omega_{c} \tau \sim 3.1 B$. The fit, especially at high fields, is also superior to the two-fluid model discussed (and found inadequate) in Ref. 6. The fit to this puddle model would be nearly perfect over the entire range of $B$ studied experimentally if $\tau$ varies by about $30 \%$ as a function of $B$. The results for $\rho_{x x}$ are independent of the sign of the charge and are thus unchanged if $f_{n} \rightarrow 1-f_{n}$.

In Fig. 2, we show the corresponding results for $\rho_{x y}(B)$. In this case, we use a field-independent $\tau$ (corresponding to $\left.\omega_{c} \tau=2.3 B\right)$. We show results for $f_{n}=0.43,0.57$, and 0.5 . $\rho_{x y}(B)$ for $f_{n}=0.43$ is equal and opposite to that for $f_{n}$ $=0.57$. In both cases, $\rho_{x y}$ varies roughly linearly with $B$ for $B$ greater than about $1 \mathrm{~T}$. At $f_{n}=0.5, \rho_{x y}=0$ for all $B$. Within the present model, this latter result is exact, and not restricted to the EMA. ${ }^{10}$

Figure 3 shows $\rho_{x x}\left(B, f_{n}\right)$ versus $f_{n}$ for several values of $B$. We use the EMA and the same parameters as in Figs. 1 and 2 (with $\omega_{c} \tau=2.3 B$ ). $\rho_{x x}$ saturates for all values of $f_{n}$ except $f_{n}=1 / 2$, for which it increases linearly with $B$. Once again, this linearity is exact, and not restricted to the EMA. ${ }^{10}$ In Fig. 4 , we show $\rho_{x y}\left(B, f_{n}\right)$ versus $f_{n}$ for several values of $B$. As can be seen, $\rho_{x y}$ changes sign at $f_{n}=1 / 2$ and approaches a constant as $f_{n}$ approaches either 1 or 0 . The magnitude of the slope $\left[d \rho_{x y} / d f_{n}\right]_{f_{n}=1 / 2}$ increases with increasing $B$ so that, at large $B$, the Hall resistivity is very close to that of the majority charge carrier.

Thus far, our numerical results have been limited to the special case where $n=p$ and $\tau_{n}=\tau_{p}$. To illustrate what happens when these assumptions are relaxed, we have carried out numerous additional calculations using the effectivemedium approximation. A representative example is shown in Fig. 5. In this case, we assume $n \neq p$ and also $\tau_{n} \neq \tau_{p}$. We also take $\sigma_{n, 0} \propto n$ and $\sigma_{p, 0} \propto p$. This implies that $\tau_{n} \propto n^{1 / 2}$ and $\tau_{p} \propto p^{1 / 2}$, which is the expected behavior for relaxation times dominated by Coulomb scattering. In addition, we choose $n / p$ and $\mu_{n} / \mu_{p}$ so as to optimize the agreement between our calculations and experiment. The resulting parameters are $n / p=1.376$ and $\mu_{n} / \mu_{p}=0.7$, with $\mu_{p}=2.3 \mathrm{~T}^{-1}$ as in our other calculations above. The plots shown in Fig. 5 are drawn at the charge neutrality point, which now lies at $f_{n}$ $=0.4209$ for this choice of $n / p$. Thus, in contrast to the examples shown in Figs. 1-4, this model gives a saturating (but large) magnetoresistance at the charge neutrality point, in agreement with experiment. The calculated Hall resistivity, however, does not vanish at this value of $f_{n}$, as shown in Fig. 5(b). In fact, for all models we have considered, the Hall resistivity changes sign at $f_{n}=1 / 2$, whatever the value of $n / p$ or $\mu_{n} / \mu_{p}$. 


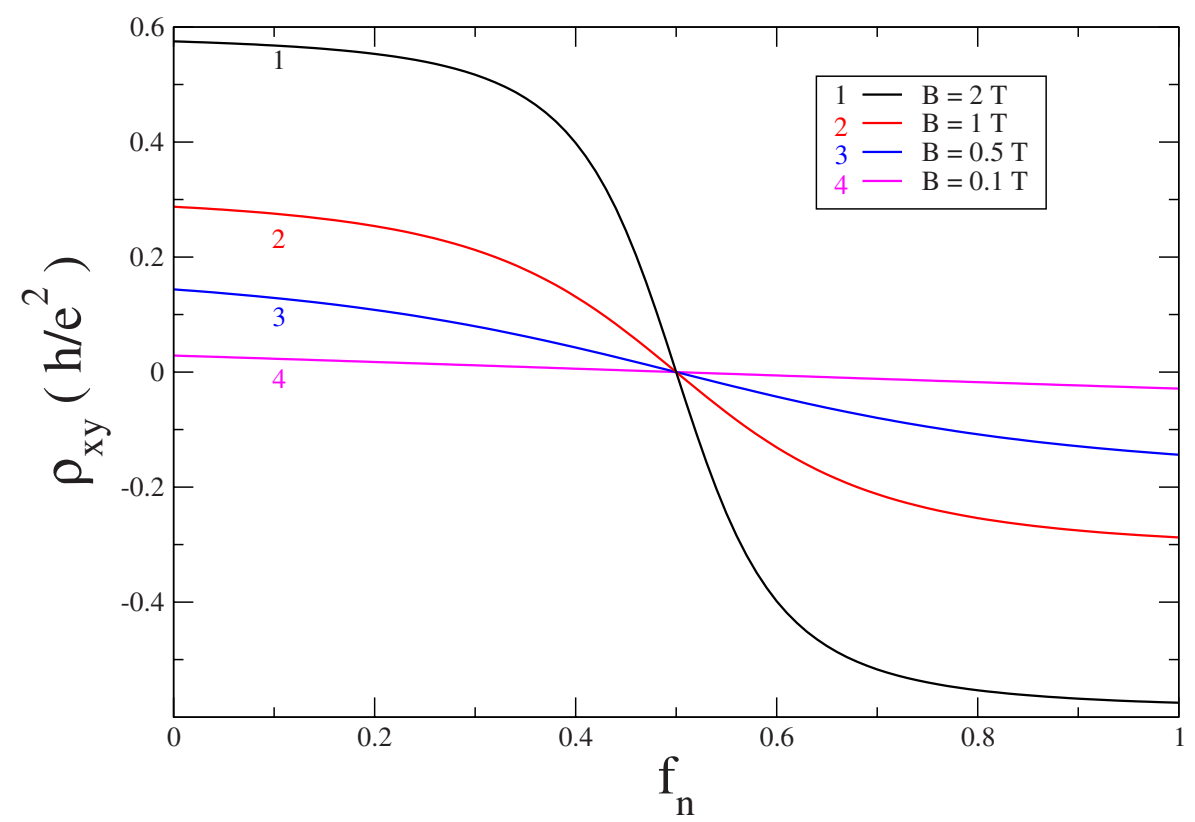

FIG. 4. (Color online) Calculated $\rho_{x y}$ as a function of $f_{n}$ for several different values of $B$ using $\mu=2.3 \mathrm{~T}^{-1}$.

\section{DISCUSSION}

\section{A. Physical origin of the large magnetoresistance and possible scaling behavior}

We turn now to a discussion of our results. First, what is responsible for the large magnetoresistance emerging from our calculations? This large magnetoresistance seems surprising because the individual $n$ and $p$ puddles have zero magnetoresistance, yet that of the composite is large. Clearly, the magnetoresistance must originate in current distortion effects. Because the conductivity tensor is inhomogeneous at finite fields, the current does not travel in a straight line through the medium in the presence of a magnetic field, but is strongly distorted by discontinuities at the boundaries between the $n$ - and $p$-type conductors. Since the current travels a longer path than it would in a homogeneous medium, the resistance should be larger. In a three-dimensional metal with a small concentration of macroscopic defects, this current distortion effect is known to produce a magnetoresistance linear in field. ${ }^{12,16}$ However, in $2 \mathrm{D}$, we have not found a simple physical argument which would show that the magnetoresistance varies linearly with $B$ at $f_{n}=f_{p}$ and saturates at other concentrations. We can say only that the linearity is precisely true in some models, as was discussed previously in Ref. 10, and also that it emerges from our effectivemedium calculations.

An intriguing question about our result is the role of the geometrical percolation threshold. In two dimensions, if one has a composite of two components ( are distributed in some symmetrical way, then the percolation threshold for either component occurs at an area fraction of $1 / 2$. That is, if the area fraction $f_{n}>1 / 2$, there is an infinite connected cluster of $n$, and if $f_{n}<1 / 2$, the connected cluster of $n$ is only finite. An analogous statement can be made about the $p$ component. If $\sigma_{n, 0}=\sigma_{p, 0}$, this geometrical percolation will have no effect on transport in our model at $B=0$ because the medium is effectively homogeneous. However, at large $B$, the two components have very different conductivities, so the percolation threshold should have a much stronger effect.

Based on the above argument, and our numerical results, we suggest that at large fields, the resistivity $\rho_{e, x x}(B)$ in our model has a singular "scaling" behavior of the form

$$
\rho_{e, x x}\left(B, f_{n}\right)=B^{c} F\left(\left|f_{n}-1 / 2\right|^{-a} / B\right),
$$

where $c$ and $a$ are suitable critical exponents which can be inferred from our numerical results. First, $\rho_{e, x x} \propto B$ at $f_{n}$ $=1 / 2$. This implies that $B^{c} F(\infty) \propto B$ or $c=1$. For $f_{n} \neq 1 / 2$, our effective-medium results show that $\rho_{e, x x}\left(B \rightarrow \infty, f_{n}\right) \propto \mid f_{n}$ $-1 /\left.2\right|^{-1}$ (this behavior can be seen in Fig. 3). This behavior, combined with $c=1$, implies that $a=1$. Thus, our effectivemedium results are consistent with Eq. (9) with $c=1$ and $a$ $=1$.

The physical basis for this scaling behavior is presumably that there are two divergent lengths in our problem at small $\left|f_{n}-1 / 2\right|$ and large $B$. The first is the linear dimension of the percolation cluster, $\xi_{p}$, which diverges near the percolation threshold. The second is a length related to the magnetic field. This length will vary as some power of $\omega_{c} \tau$. The ratio of these two lengths should determine the behavior of $\rho_{e, x x}\left(B, f_{n}\right)$ near a hypothetical high-field critical point at $f_{n}$ $=1 / 2$ and $B=\infty$. However, we emphasize that this scaling behavior is rather speculative since we can verify it only within the effective-medium approximation.

\section{B. Connection to experiments in graphene}

The present model agrees well with the measured values of $\rho_{x x}(B)$ in graphene. However, it is based on certain assumptions whose validity for graphene we now discuss. One assumption is that graphene can be treated as a macroscopically inhomogeneous assembly of puddles, each with its own conductivity tensor. The scanning probe images shown in Ref. 5 suggest that the carrier density varies appreciably over a distance of perhaps $0.2 \mu$. Using the above density estimates, the number of charge carriers in a puddle of linear 

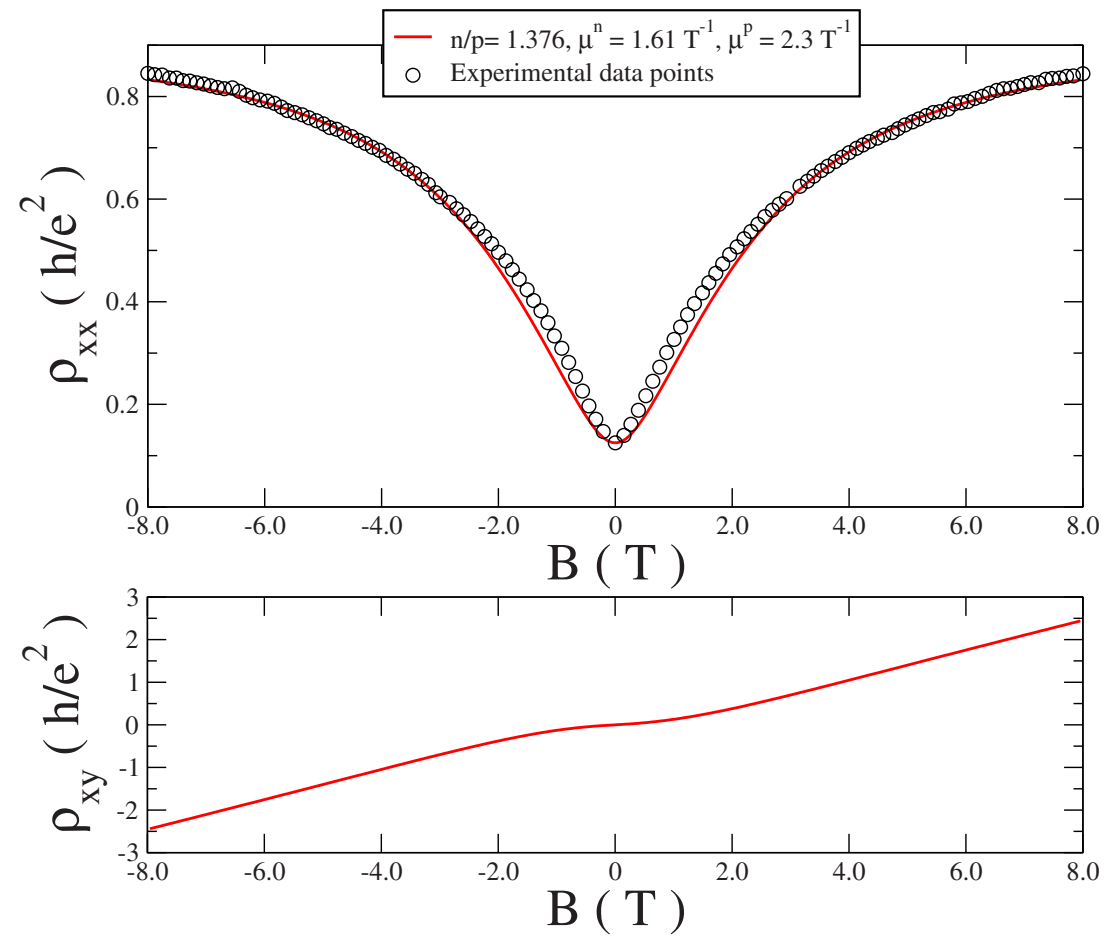

FIG. 5. (Color online) Calculated and measured $\rho_{x x}(B)$ and calculated $\rho_{x y}(B)$ with $B$ in Tesla. The calculations are carried out using different assumptions than Figs. 1 and 2. Specifically, we assume that $n / p=1.376, \quad \mu_{n} / \mu_{p}$ $=0.7$, and $\mu_{p}=2.3 \mathrm{~T}^{-1}$. We also assume that $\sigma_{n, 0} \propto n$ and $\sigma_{p, 0} \propto p$. The calculations are carried out at the charge neutrality point, $f_{n}$ $=0.4209$. In both panels, the full curves are the calculated values, while the circles in the top panel represent experimental data from Ref. 6.

dimension $0.2 \mu$ would be $\sim 25$. This size is rather small to be treated macroscopically. On the other hand, a more reasonable definition of a "puddle" might be a region where the charge carriers were all of one sign. Judging from the images, a typical linear dimension of such a region would be larger than $0.2 \mu$, perhaps $0.5 \mu-1 \mu$, and would contain $\sim 500$ carriers. This is probably large enough to describe each puddle by its own macroscopic conductivity provided that the mean free path $\Lambda$ is less than $1 \mu$. In fact, experimentally measured $\Lambda$ for both suspended and non-suspended graphene sheets with typical carrier densities considered here $\left(6 \times 10^{14} \mathrm{~m}^{-2}\right)$ are less than $0.1 \mu$ for reasonable values of temperature. ${ }^{17}$ Thus, treating the puddle mixture macroscopically is probably appropriate in the case of some disordered samples of graphene, and the results seem to agree with experiment.

Another point, as can be seen from Fig. 3, is that the $\rho_{x x}\left(B, f_{n}\right)$ saturates only if $f_{n} \neq 1 / 2$. If $n=p$, then $f_{n} \neq 1 / 2$ would imply a net charge imbalance produced by a suitable gate voltage. However, if $n \neq p$, we can still have a saturating magnetoresistance and no net charge imbalance, as can be seen in Fig. 5. Such saturation seems to be observed in experiments. ${ }^{6}$ As can be seen from Figs. 2, 4, and 5, even if there is no charge imbalance, a value of $f_{n} \neq 1 / 2$ would still lead to a nonzero $\rho_{x y}(B)$. It would be of interest if $\rho_{x y}(B)$ could be measured and compared to the values needed for the present model to agree with experiment.

Third, the present model treats the electron dynamics semiclassically and thus does not take into account the quantum Hall effect (QHE), which is seen at sufficiently high fields. ${ }^{1,15,18}$ Typically, the QHE will become visible when the spacing between the Landau levels is large compared to $k_{B} T$. This can occur even at room temperature in graphene. ${ }^{18}$ If the QHE becomes important, the present semiclassical model would need to be modified.
A fourth point is that we have carried out our calculations within the EMA, which may introduce some error. In this regard, it would be of some interest if one could calculate the magnetotransport of a model of $n$ and $p$ puddles using a more exact procedure, such as a suitably defined network of random impedance matrices. Such a calculation has previously been carried out for a somewhat different model of $n$ and $p$ carriers $^{19}$ and could, in principle, be extended to the present problem.

Finally, we obtain the best fits to experiment if we assume a weakly magnetic-field-dependent relaxation time, as described above. Such field dependence could be reasonable, but it would be useful to have a model which explicitly produces a magnetic-field-dependent $\tau$.

We find that our results are quite insensitive to slight changes in the parameters or other features of the model. For example, Fig. 1 suggests that $\rho_{x x}$ changes only slightly, but not dramatically, when $\tau$ is varied by $\sim 30 \%$. Also, we have recalculated $\rho_{e, x x}(B, T)$ without the assumption that the electrons and holes have equal mobilities. Even if the mobilities are different, we find that $\rho_{x x}(B)$ still varies linearly with $B$ at $f_{n}=0.5$ and saturates at other values of $f$. Another change in our model is suggested by that fact that the carrier density in graphene must be a continuous function of position rather than being simply bimodal as postulated in our model. To check the effects of a non-bimodal distribution, we have repeated our calculations assuming four types of puddles, two $n$ and two $p$, with two different densities each of electrons and holes. Once again, we find that the resulting $\rho_{x x}\left(B, f_{n}\right)$ depends primarily on $f_{n}$ and $\tau$, and not on the presence of two types of $n$ and of $p$ puddles. Finally, we have considered the case of a three-component composite, made up of $n$ type, $p$ type, and insulating regions. Here, once again, we find that, for a small insulating areal faction $(\sim 0.1)$, we obtain linear magnetoresistance if $f_{n}=f_{p}$ and saturating magnetoresistance 
otherwise, similar to the case of no insulating regions. Thus, our results are not much affected by small modifications in our model.

To summarize, we have calculated the magnetoresistance and Hall resistivity for a semiclassical model of graphene, on the assumption that it is a mixture of $n$-type and $p$-type puddles, and using the correct form of the band structure near the Dirac points. The resulting magnetoresistance is in good agreement with experiment provided that the areal fractions of $n$ - and $p$-type puddles are slightly different and that the relaxation time is weakly magnetic field dependent. Fur- ther confirmation of the model could be obtained if the measured Hall resistivity were compared to that computed from this model.

\section{ACKNOWLEDGMENTS}

Funding for this research was provided by the Center for Emergent Materials at the Ohio State University, an NSF MRSEC (Award No. DMR-0820414). We thank N. J. Harmon for valuable conversations.
${ }^{1}$ K. S. Novoselov, A. K. Geim, S. V. Morozov, D. Jiang, M. I. Katsnelson, I. V. Grigorieva, S. V. Dubonos, and A. A. Firsov, Nature (London) 438, 197 (2005).

${ }^{2}$ F. Miao, S. Wijeratne, Y. Zhang, U. C. Coskun, W. Bao, and C. N. Lau, Science 317, 1530 (2007).

${ }^{3}$ J. H. Chen, M. Ishigami, C. Jang, D. R. Hines, M. S. Fuhrer, and E. D. Williams, Adv. Mater. (Weinheim, Ger.) 19, 3623 (2007).

${ }^{4}$ S. Adam, E. H. Hwang, V. M. Galitski, and S. D. Sarma, Proc. Natl. Acad. Sci. U.S.A. 104, 18392 (2007).

${ }^{5}$ J. Martin, N. Akerman, G. Ulbricht, T. Lohmann, J. H. Smet, K. Von Klitzing, and A. Yacoby, Nat. Phys. 4, 144 (2008).

${ }^{6}$ S. Cho and M. S. Fuhrer, Phys. Rev. B. 77, 081402(R) (2008).

${ }^{7}$ See, e.g., N. W. Ashcroft and N. D. Mermin, Solid State Physics, (Saunders, Orlando, FL, 1976), Eq. (12.42).

${ }^{8}$ K. I. Bolotin, K. J. Sikes, Z. Jiang, M. Klima, G. Fudenberg, J. Hone, P. Kim, and H. L. Stormer, Solid State Commun. 146, 351 (2008).

${ }^{9}$ D. Stroud, Phys. Rev. B 12, 3368 (1975).

${ }^{10}$ V. Guttal and D. Stroud, Phys. Rev. B 71, 201304(R) (2005).

${ }^{11}$ See, e.g., D. J. Bergman and D. Stroud, Solid State Phys. 46,
147 (1992) for a discussion of this point.

${ }^{12}$ D. Stroud and F. P. Pan, Phys. Rev. B 13, 1434 (1976).

${ }^{13}$ See, e.g., P. J. Reynolds, H. E. Stanley, and W. Klein, Phys. Rev. B 21, 1223 (1980).

${ }^{14}$ See, e.g., C. Berger, Z. M. Song, X. B. Li, X. S. Wu, N. Brown, C. Naud, D. Mayou, T. B. Li, J. Hass, A. N. Marchenkov, E. H. Conrad, P. N. First, and W. A. de Heer, Science 312, 1191(2006).

${ }^{15}$ Z. Jiang, E. A. Henriksen, L. C. Tung, Y.-J. Wang, M. E. Schwartz, M. Y. Han, P. Kim, and H. L. Stormer, Phys. Rev. Lett. 98, 197403 (2007).

${ }^{16}$ J. B. Sampsell and J. C. Garland, Phys. Rev. B 13, 583 (1976).

${ }^{17}$ X. Du, I. Skachko, A. Barker, and E. Y. Andrei, Nat. Nanotechnol. 3, 491 (2008).

${ }^{18}$ K. S. Novoselov, Z. Jiang, Y. Zhang, S. V. Morozov, H. L. Stormer, U. Zeitler, J. C. Maan, G. S. Boebinger, P. Kim, and A. K. Geim, Science 315, 1379 (2007).

${ }^{19}$ M. M. Parish and P. B. Littlewood, Nature (London) 426, 162 (2003). 